routers. A wireless router serves as a base station, providing wireless connections to any Wi-Fi-enabled devices within range of the router's wireless signal. This includes laptops, tablets, smart phones, and other wireless devices, such as smart appliances and smart home controllers. Wireless routers often connect to a cable modem or other Internet-connected device to provide Internet access to connected devices.

LANs and WLANs can be merged together using a bridge that connects the two networks. Many wireless routers also include Ethernet ports, providing connections for a limited number of wireless devices. In most cases, wireless routers act as a bridge, merging the Ethernet and Wi-Fi-connected devices into the same network. This allows wired and wireless devices to communicate with each other through a single router.

Wireless bounded breadth arrangement Technology is created from about 2 aspects one is arrangement interface agenda (NIC) and addition is admission purpose (AP). NIC could be an adapted with aural the laptop through the NIC laptop affiliated to an altered laptop and we will forward and accept the information. AP is wireless action NIC affiliated to the AP and so ability forwards to an atomic one another.

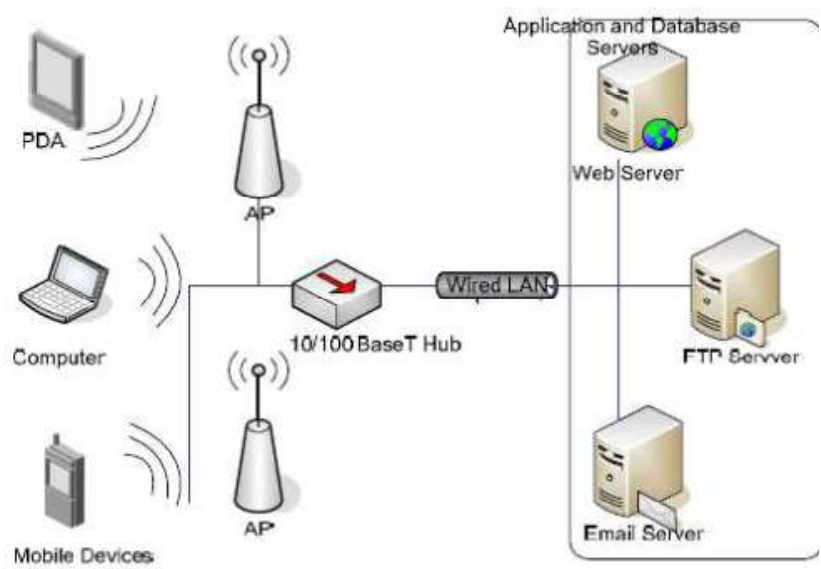

Fig 1: WLAN becomes very important in the voice based ad-hoc networks, which requires the dedicated connections for the exchange of the voice data over WLAN. The creation of the adaptive ad-hoc network routing solution takes the perfectly layered amalgamation of the genetic programming based routing solution along with the connection integrity assurance model in order to realize the robust ad-hoc routing algorithm.

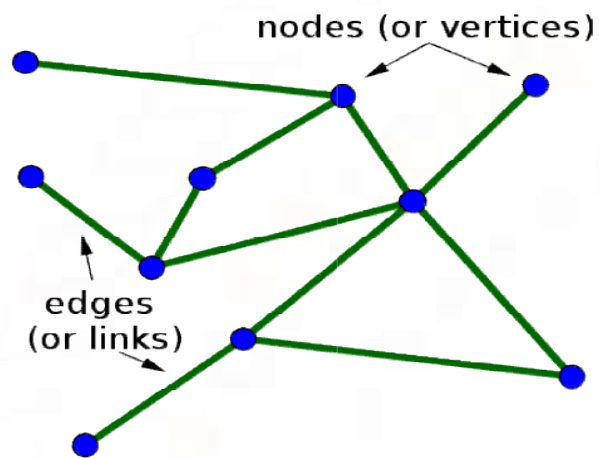

Fig 2: Nodes and Edges

\section{RESULT ANALYSIS}

The results have been obtained from the proposed model simulation for the realization of the robust and flexible routing algorithm. The proposed model is checked under the Blackhole attack, DDOS and Selective Jamming attack. The major parameters of PDR (packet delivery ratio), Network Load, Throughput and transmission (end-to-end) delay have been analyzed under this section.

\begin{tabular}{|c|c|}
\hline Time & Delay ( $\mathrm{ms})$ \\
\hline 10 & 30 \\
\hline 20 & 56 \\
\hline 30 & 43 \\
\hline 40 & 80 \\
\hline
\end{tabular}

Table 1.1 Delay under black hole attack

\begin{tabular}{|c|c|}
\hline Time & $\begin{array}{c}\text { Network } \\
\text { Load(KBPS) }\end{array}$ \\
\hline 10 & 0.048189356 \\
\hline 20 & 0.291080506 \\
\hline 30 & 0.83401428 \\
\hline 40 & 1 \\
\hline
\end{tabular}

Table 1.2 Network Load under black hole attack and connection integrity assurance algorithm. The new combination is capable of adding the higher level of security for the prevention of the connectivity holes and the fake route injections in the given network. The smart path selection across the multipath network 
International Journal of Trend in Scientific Research and Development (IJTSRD) ISSN: 2456-6470

\begin{tabular}{|c|c|}
\hline Time & Data Loss (KB) \\
\hline 10 & 1.02 \\
\hline 20 & 2.75 \\
\hline 30 & 3.69 \\
\hline 40 & 4.75 \\
\hline
\end{tabular}

Table 1.3 Data Loss under black hole attack

\begin{tabular}{|c|c|}
\hline Time & PDR \\
\hline 10 & $15 \%$ \\
\hline 20 & $38 \%$ \\
\hline 30 & $62 \%$ \\
\hline 40 & $94 \%$ \\
\hline
\end{tabular}

Table 1.4 Packet delivery Ratio under black hole attack

\begin{tabular}{|c|c|}
\hline Time & Throughput(KBPS) \\
\hline 10 & 8.3 \\
\hline 20 & 73 \\
\hline 30 & 100 \\
\hline 40 & 142 \\
\hline
\end{tabular}

Table 1.5 Throughput under black hole attack

\section{Reading Under DDOS Attack}

\begin{tabular}{|c|c|}
\hline Time & Data Loss (KB) \\
\hline 10 & 1.616 \\
\hline 20 & 2.15 \\
\hline 30 & 2.872 \\
\hline 40 & 5.8 \\
\hline
\end{tabular}

Table 1.6 Data Loss under DDOS attack

\begin{tabular}{|c|c|}
\hline Time & PDR \\
\hline 10 & $20 \%$ \\
\hline 20 & $36 \%$ \\
\hline 30 & $55 \%$ \\
\hline 40 & $56 \%$ \\
\hline
\end{tabular}

Table 1.7 Packet delivery Ratio under DDOS attack

\begin{tabular}{|c|c|}
\hline Time & Throughput(KBPS) \\
\hline 10 & 12 \\
\hline 20 & 36 \\
\hline 30 & 42 \\
\hline 40 & 62 \\
\hline
\end{tabular}

Table 1.8 Throughput under DDOS attack

\begin{tabular}{|c|c|}
\hline Time & Network Load \\
\hline 10 & 1.66 \\
\hline 20 & 3.32 \\
\hline 30 & 5.15 \\
\hline 40 & 9.77 \\
\hline
\end{tabular}

Table 1.9 Network Load under DDOS attack

\begin{tabular}{|c|c|}
\hline Time & Delay \\
\hline 10 & 3 \\
\hline 20 & 3 \\
\hline 30 & 4.8 \\
\hline 40 & 5.66 \\
\hline
\end{tabular}

Table 1.10 Delay under DDOS attack

\section{Reading under Jamming Attack}

\begin{tabular}{|c|c|}
\hline Time & $\begin{array}{c}\text { Data Loss } \\
\text { (KB) }\end{array}$ \\
\hline 10 & 0.77 \\
\hline 20 & 0.87 \\
\hline 30 & 0.94 \\
\hline 40 & 1 \\
\hline
\end{tabular}

Table 1.11 Data Loss under DDOS attack

\begin{tabular}{|c|c|}
\hline Time & PDR \\
\hline 10 & $55 \%$ \\
\hline 20 & $78 \%$ \\
\hline 30 & $90 \%$ \\
\hline 40 & $91 \%$ \\
\hline
\end{tabular}

Table 1.12 Packet delivery Ratio under DDOS attack

\begin{tabular}{|c|c|}
\hline Time & Throughput(KBPS) \\
\hline 10 & 58 \\
\hline 20 & 146 \\
\hline 30 & 147 \\
\hline 40 & 190 \\
\hline
\end{tabular}

Table 1.13 Throughput under DDOS attack

\begin{tabular}{|c|c|}
\hline Time & Network Load \\
\hline 10 & 1.93 \\
\hline 20 & 3.3 \\
\hline 30 & 4.4 \\
\hline 40 & 4.35 \\
\hline
\end{tabular}

Table 1.14 Network Load under DDOS attack 
International Journal of Trend in Scientific Research and Development (IJTSRD) ISSN: 2456-6470

\begin{tabular}{|c|c|}
\hline Time & Delay \\
\hline 10 & 14 \\
\hline 20 & 15 \\
\hline 30 & 15 \\
\hline 40 & 30 \\
\hline
\end{tabular}

Table 1.15 Delay under DDOS attack

\section{Delay Analysis}

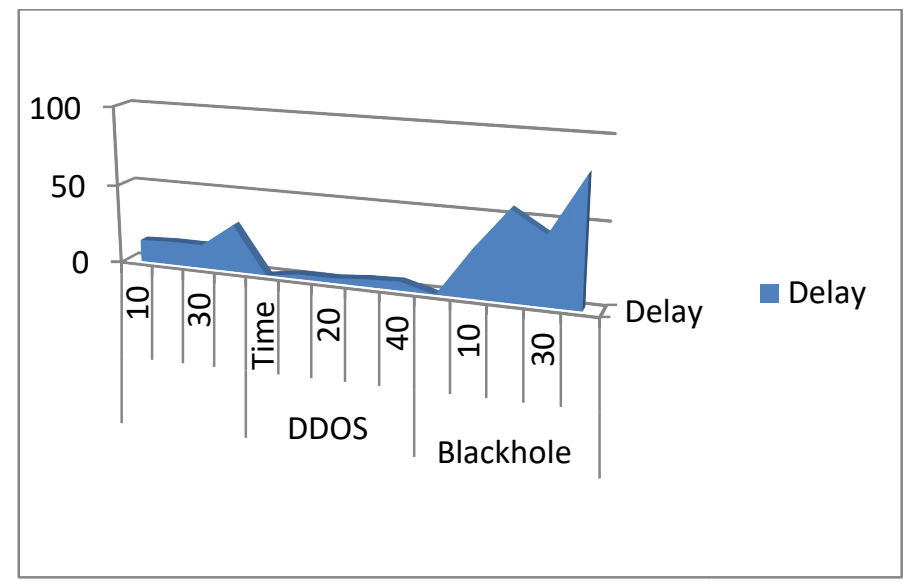

Fig 3: Analysis of Delay under all the attacks

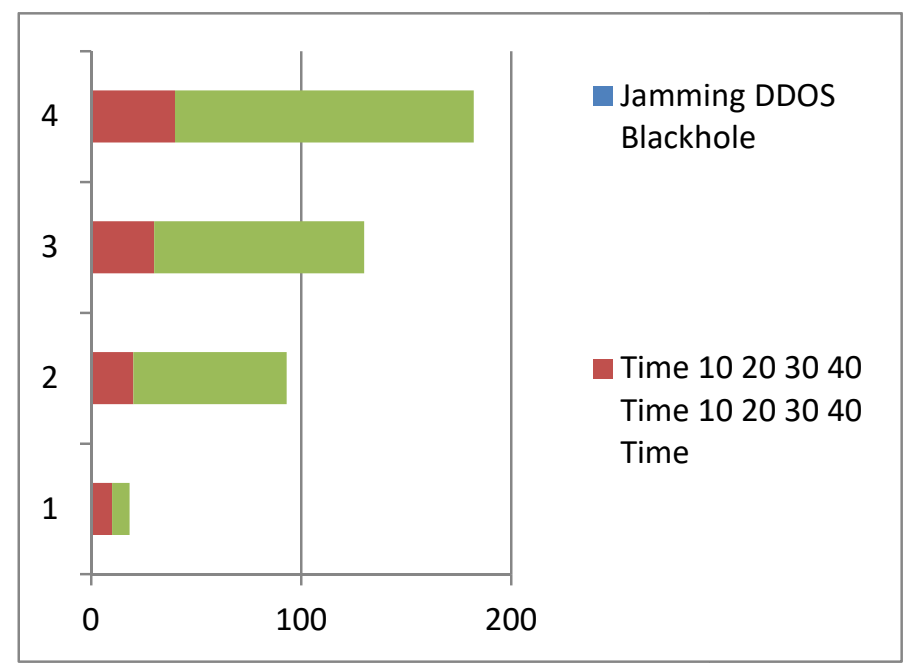

Fig 4: Throughput Analysis under all attacks.

Packet Delivery Rate: Packet delivery ratio factor evaluates the percentage of the successfully delivered packets among the given network or link in the given time ( 1 second in our case). The PDR shows the rising trend in the following graphs, which elaborates the rising network ability with each passing second, whereas the stability or straight line after $10^{\text {th }}$ second shows the performance of the fully converged network, which is communicating on the nearly constant speed to deliver the packets among the given networks.
Energy Consumption: The energy consumption has been observed in the Joules for each node-to-node connection, also denoted a path in the network. In this simulation, the initial energy is assigned to each of the node during the beginning of the simulation, which is tracked and reduced with the transmission, receive and routing (for intermediate nodes only) phase. The remaining energy in the end is recorded in the following graph, where the steep falling curve has been observed in contrast to the figure 1.1, which shows the energy consumption with rising number of packets, whereas after nearly $7^{\text {th }}$ second, the curve remains nearly constant to show the network convergence.

\section{REFERENCES}

[1] A wireless bounded breadth arrangement Association (2002). "Wireless Networking Standards and Organizations", WLAN Resource Center, April 17, 2002

[2] Wireless bounded breadth arrangement Medium Admission administration (1999) And Physical Band (PHYJ. Specification,

[3] A. Wood and J. Stankovic (2002). Denial of account in assay aspect network. IEEE pc,

[4] Taewoo Kwon, Emre Ertin, Anish Arora (2012) Reproducing constant wireless agreement achievement beyond environments, ad-lib Networks, ten (2012) 696“708, Elsevier

[5] Benot Latre, Bart Braem, Ingrid Moerman Chris Blondia, Piet Demeester ( 2011) A assay on wireless physique amplitude networks, Wireless Arrangement (2011) 17:1вЂ“18 ， DOI 10.1007/s11276-010-0252-4.

[6] Sung-Hwa Lim, Young-Bae blow, Cheolgi Kim and Nitin H. Vaidya (2011) appearance and accomplishing of multicasting for multi-channel multi-interface wireless cobweb networks, Wireless Arrangement (2011) 17:955в Ђ“972.

[7] Petrioli, Chiara, et al. "ALBA-R: Load-balancing geographic routing around connectivity holes in wireless sensor networks." Parallel and Distributed Systems, IEEE Transactions on 25.3 (2014): 529539.

[8] Y. Zhao, Q. Zhang, Y. Chen, and W. Zhu, "Hop ID Based Routing in Mobile Ad Hoc Networks," Proc. IEEE 13th Int'l Conf. Network Protocols (ICNP '05), pp. 179-190, Nov. 2005. 
International Journal of Trend in Scientific Research and Development (IJTSRD) ISSN: 2456-6470

[9] S. Basagni, M. Nati, and C. Petrioli, "Localization Error-Resilient Geographic Routing for Wireless Sensor Networks," Proc. IEEE GLOBECOM, pp. 1-6, Nov./Dec. 2008.

[10] Xu, Jiu-qiang, et al. "Study on WSN topology division and lifetime." Computer Science and Automation Engineering (CSAE), 2011 IEEE International Conference on. Vol. 1. IEEE, 2011. 